\title{
Determination of the laser-induced damage threshold of polymer optical fibers
}

Kiedrowski, K., Thiem, J., Jakobs, F., Kielhorn, J., Balasa, I., et al.

K. Kiedrowski, J. Thiem, F. Jakobs, J. Kielhorn, I. Balasa, D. Kracht, D. Ristau, "Determination of the laser-induced damage threshold of polymer optical fibers," Proc. SPIE 10805, Laser-Induced Damage in Optical Materials 2018: 50th Anniversary Conference, 108052C (16 November 2018); doi: $10.1117 / 12.2500336$ 


\title{
Determination of the laser-induced damage threshold of polymer optical fibers
}

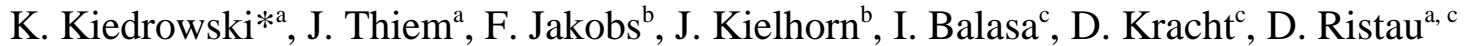 \\ ${ }^{a}$ Institute of Quantum Optics, Leibniz University Hannover, Welfengarten 1, 30167 Hannover, \\ Germany; \\ ${ }^{b}$ Institute of High Frequency Technology, TU Braunschweig, Schleinitzstraße 22, 38106 \\ Braunschweig, Germany; \\ ${ }^{c}$ Laser Zentrum Hannover e.V., Hollerithallee 8, 30419 Hannover, Germany
}

\begin{abstract}
Investigating the properties of manufactured polymer optical fibers is essential to determine proper areas of application. Using pulsed laser radiation, especially with respect to laser activity in optical fibers, the maximum acceptable transmittable energy without inducing damage is of particular interest. Therefore, this work is related to laser-induced damage in polymer optical fibers at a wavelength of $532 \mathrm{~nm}$ and a pulse duration of $7.3 \mathrm{~ns}$. In particular, the influence of the coupling condition on the transmittable pulse energy and the damage behavior applying an R-on-1 test procedure are analyzed in this study. The obtained results give information about the long-term behavior and will be used to optimize the manufacturing process.
\end{abstract}

Keywords: polymer optical fiber, laser-induced damage, SLOT, PMMA

\section{INTRODUCTION}

The properties of polymer optical fibers (POFs) differ in many aspects from those of glass fibers. POFs stand out by a low net weight and a high mechanical flexibility with regards to bending, compression and stretching. However, disadvantages such as a high attenuation [1] and a low optical radiation resistivity limit the applicability of the fiber. In this work the laser-induced damage of POFs at a wavelength of $532 \mathrm{~nm}$ and a pulse duration of $7.3 \mathrm{~ns}$ is studied. For this investigation R-on-1 tests are performed using 1000 pulses for each energy level until catastrophic damage occurs. Three different damage definitions will be distinguished and evaluated with regard to possible causes. Gebauer et al. [2] investigated the influence of two coupling lenses with different focal length on the laser-induced damage threshold (LIDT) of glass fibers and observed no significant impact on the transmittable pulse energy. This behavior is investigated in this study for polymer optical fibers.

The laser-induced damage is visualized using scanning laser optical tomography (SLOT) serving as an ex-situ detection method. This ensures the determination of the damage location in the radial direction and provides information on the damage morphology, especially with respect to the three defined damage definitions.

\section{FIBER PREPARATION AND EXPERIMENTAL SETUP}

\subsection{Properties and preparation of the polymer optical fibers}

The polymer optical fibers used in this investigation were manufactured by our project partner the TU Braunschweig. The corresponding properties of the core and cladding material are listed in table 1 . After cutting the fiber into $20 \mathrm{~cm}$ long pieces, both facets were prepared by hand using polishing sheets with a $3 \mu \mathrm{m}, 1 \mu \mathrm{m}$ and $0.3 \mu \mathrm{m}$ grit. Furthermore, a cotton bud and isopropanol are used before each measurement to remove impurities on the fiber facets originating from the polishing process.

The attenuation of the polymer optical fibers (figure 1) was determined using the cutback method [3]. The peaks occurring in the attenuation spectrum are mostly caused by the $\mathrm{C}-\mathrm{H}$ stretching and bending vibrations (e.g. fifth overtone $\mathrm{C}-\mathrm{H}$ stretching vibration at $736 \mathrm{~nm}$ [4]). It is expected that for a $20 \mathrm{~cm}$ long fiber the attenuation leads to a transmission loss of around $9.3 \%$. Moreover, coupling losses and Fresnel losses add up lowering the transmission efficiency. Fresnel losses in case of normal incidence of approximately $4 \%$ at the front and back interface reduce the output transmission to $92.16 \%$. In this study transmission efficiencies of approximately $(77 \pm 6) \%$ were achieved on average. Thus, the average coupling loss amounts to about 5 to $6 \%$. However, the following values of the laser pulse energy refer to the incident pulse energy, on the entrance facet.

*kiedrowski@iqo.uni-hannover.de; phone+49 5112788 363; fax +49 5112788100

Laser-Induced Damage in Optical Materials 2018: 50th Anniversary Conference, edited by Christopher Wren Carr,

Gregory J. Exarhos, Vitaly E. Gruzdev, Detlev Ristau, M.J. Soileau, Proc. of SPIE Vol. 10805, 108052C

(C) 2018 SPIE $\cdot$ CCC code: $0277-786 X / 18 / \$ 18 \cdot$ doi: $10.1117 / 12.2500336$ 
Table 1. Properties of polymer optical fibers used in this investigation.

\begin{tabular}{|l|l|}
\hline Material core/cladding & PMMA/404F acrylat \\
\hline Diameter core/fiber & $486 / 500 \mu \mathrm{m}$ \\
\hline $\begin{array}{l}\text { Refractive index } \\
\text { core/cladding @ 466 nm }\end{array}$ & $1.498 / 1.42$ \\
\hline Numerical aperture & 0.48 \\
\hline V-parameter & 1378 \\
\hline Attenuation @ 532 nm & $2131 \mathrm{~dB} / \mathrm{km}$ \\
\hline
\end{tabular}

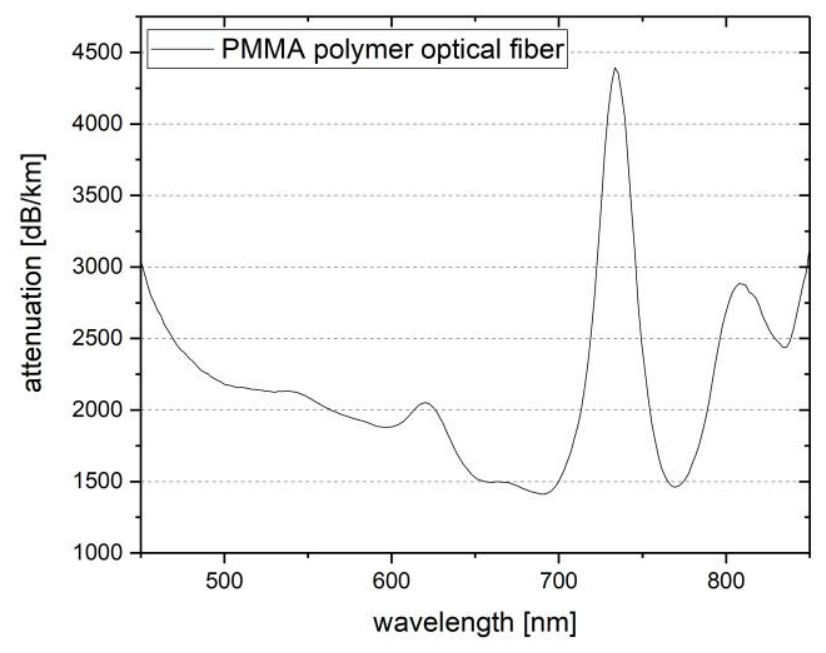

Figure 1. Attenuation of the polymer optical fibers used in this investigation determined by the cutback method.

\subsection{Experimental setup and damage definitions}

The experimental setup used in this investigation is shown in figure 2. The pulsed radiation at the frequency doubled wavelength of $532 \mathrm{~nm}$ of a Nd:YAG laser with a pulse duration of $7.3 \mathrm{~ns}$ at a repetition frequency of $10 \mathrm{~Hz}$ was used to investigate the transmittable pulse energy. Monitored by a photodiode (PD1) calibrated with the pyroelectric energy sensor ES111C, the pulse energy can be adjusted using a polarizer and a half wave plate serving as a variable attenuator. The diameter of the photodiodes active area is $9.8 \mathrm{~mm}$ and a neutral density filter is used adjusting the measured signal. The entrance facet of the fiber pieces is located behind the focus plane of the lens (L). In this investigation, three different lenses with focal lengths of $200 \mathrm{~mm}, 500 \mathrm{~mm}$ and $1000 \mathrm{~mm}$ were used for coupling into the fiber. A 3-axis stage is used to align the fiber with the optical axis of the laser beam maximizing the transmission output of the fiber by minimizing coupling losses. The fiber is placed behind the beam focus so that the effective beam diameter at the fiber facet is $185 \mu \mathrm{m}$. For this alignment, a second photodiode (PD2 identically in construction with PD1) with a neutral density filter is placed at the exit facet of the fiber measuring the transmitted energy. Moreover, this photodiode serves as an in-situ laser-induced damage detector. It is expected that a laser-induced damage along the fiber creates scattering centers, thus increasing optical losses and lowering the ratio between the two photodiodes (PD2/PD1). During the measurement, a CCD camera monitors the scattered light along the fiber and enables the in-situ determination of the damage location along the fiber. 


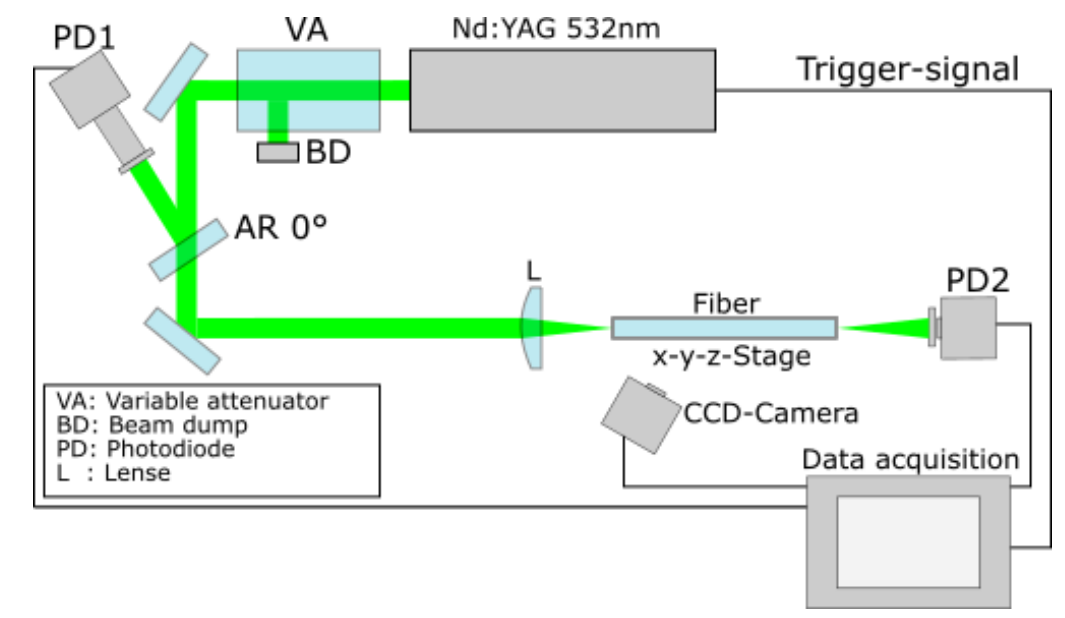

Figure 2. Measurement setup used for the determination of the transmittable pulse energy through a polymer optical fiber.

Measuring the signal of the photodiode (PD2) while placed between the lens and fiber and calculating the ratio with the fiber output signal the transmission efficiency can be determined for each measurement. Moreover, the signal ratio of the photodiodes was calculated for different pulse energies to check the linearity between both detectors. In the operative range the standard deviation of the signal ratios of eight different pulse energies was $1.2 \%$ with a maximum difference of $3.5 \%$. Furthermore, the dependency of the beam diameter at the detector on the photodiode signal was investigated. PD2 was placed at six positions between coupling lens and fiber. Thus, the illumination of the active area was varied between 30 and $80 \%$, whereby the measured maximum voltage showed a standard deviation of mean measurement values of $0.7 \%$.

The determination of the transmittable pulse energy was done performing R-on-1 tests. For each energy level a number of 1000 pulses and an increase in the pulse energy of approximately $15 \%$ with regard to the previous level was chosen. The damage tests were conducted referring to three different damage definitions:

- Low loss scatter center (LLSC): first time during the R-on1-test at which a scatter center formed along the fiber and is detected with the CCD-camera with a low relative transmission loss of less than $1 \%$

- Minor transmission loss (MTL): first relative transmission loss greater than $1 \%$

- Catastrophic damage: rapid loss in transmission of at least $10 \%$

Typically, the MTL occurred within 100 to 500 applied pulses, whereas catastrophic damage was observed within the first 10 pulses.

\section{EXPERIMENTAL RESULTS}

\subsection{Overview of the different damage events}

The formation and growth of the laser-induced damages with increasing pulse energy is shown in figure 3 .

Along the fiber scattered light is detectable with the CCD camera while transmitting pulses with a low energy through the fiber indicating impurities and defects in the fiber core and/or core-cladding-transition (fig.3 (1)). When the pulse energy is increased a scatter center forms along the fiber (fig.3 (2)). The transmission loss caused by such a scattering center can vary. Therefore, the first two definitions of damage in section 2.2 coincide for some fiber pieces. For higher pulse energies the formed scatter center might expand thus decreasing the transmission signal even more. Also more scatter centers might form along the fiber at unpredictable positions increasing the optical losses (3\& 4$)$. When the pulse energy is at a critical level, a catastrophic damage is induced within the first few centimeters of the fiber ( 4 bottom right). 

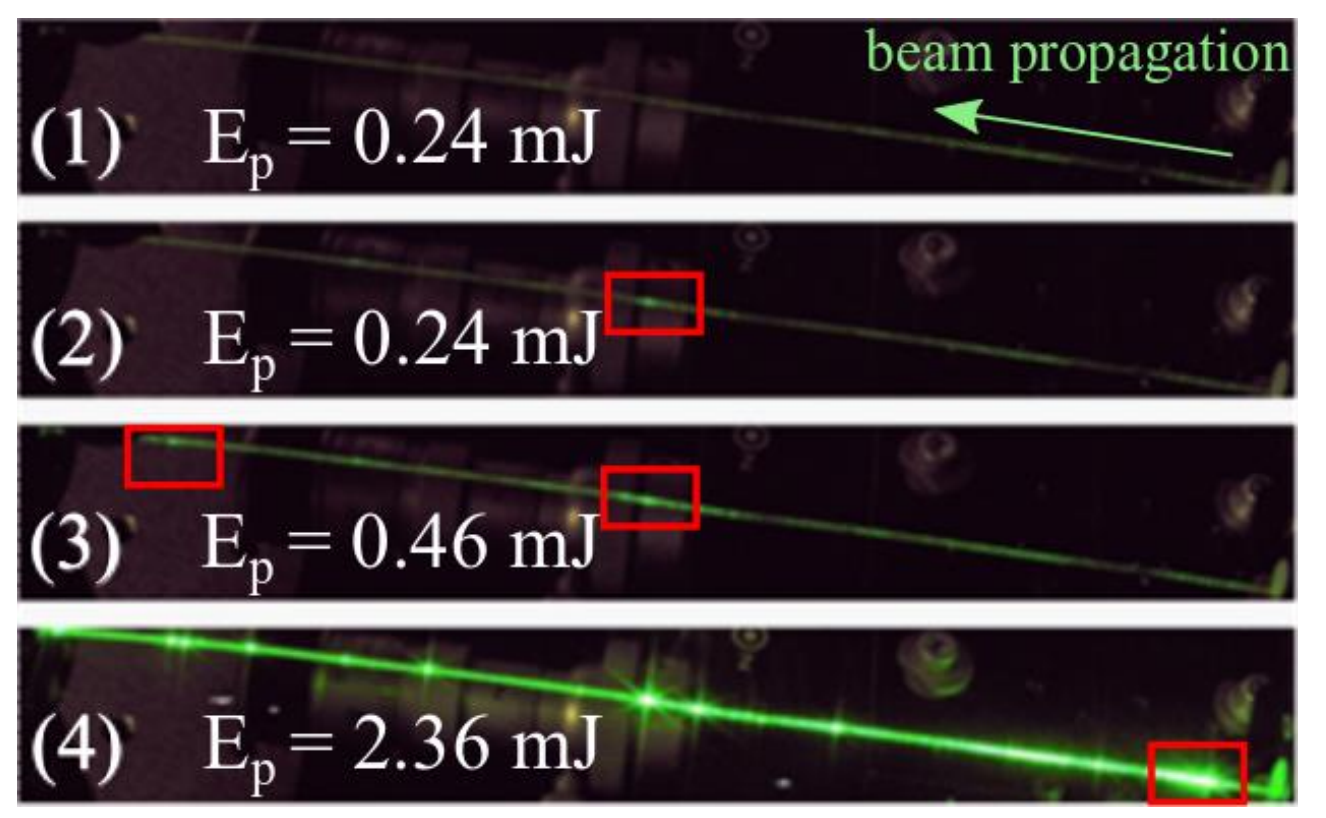

Figure 3. Damage along the fiber occurring using three different definitions. (1) no laser-induced damage, scattered light indicates impurities and defects; (2) LLSC; (3 \& 4) MTL; (4) catastrophic damage can been seen in the bottom right.

In figure 4 the locations of the laser-induced damages along the fiber are presented for the three different focal lengths and two of the damage definitions mentioned before. The position of the first damages occurring for MTL usually correspond with the location of the first detected scatter centers (LLSC) and is therefore not specifically shown in the diagram. Catastrophic damage always arises within the first few centimeters of the fiber. As mentioned before in the definitions, catastrophic damage can be described by a comparatively rapid loss in transmission within a few pulses, whereas MTL happens within several hundreds of pulses. The wide range of damage locations along the fiber, and the low loss in transmission caused by the formation of a scattering center probably indicates an induced damage of an already existing defect in the core-cladding-transition. The difference of the morphologies of these defects is precisely described in section 3.3. In addition, a significant dependence on the transmittable pulse energy on the damage position along the fiber was not observed for each damage definition.

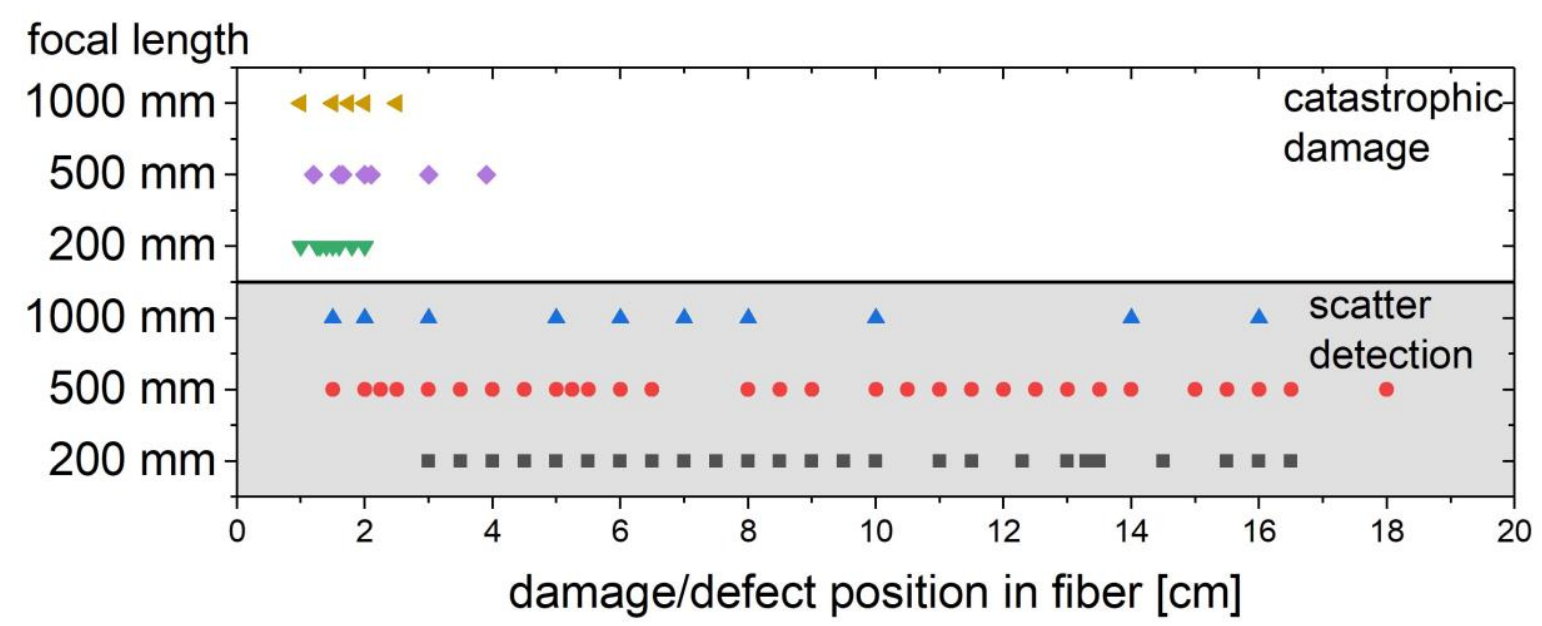

Figure 4. Positions of scattering centers and catastrophic damage along the fiber for three different focal lengths of the focusing lens. The fiber mount prevents a detection of scattered light within the last $1-2 \mathrm{~cm}$ thus the data is not shown here. 


\subsection{Transmittable pulse energy}

The laser-induced damage threshold is defined as the maximum transmittable pulse energy that does not induce damage. It is determined for each focal length by plotting the damage probability for each pulse energy. The damage probability is calculated using a cumulative approach [5] by dividing the numbers of fibers damaged up to a specific pulse energy by the total number of fibers tested. A distinction was made between the different damage definitions and the different focal lengths of the focusing lens. The intersection of a linear fit with the $\mathrm{x}$-axis determines the transmittable pulse energy at which no damage is observed (figure 5 a)).

a)

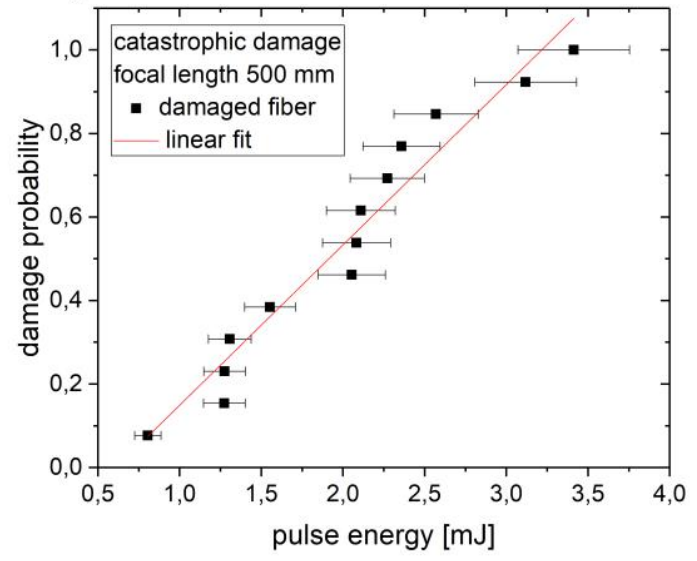

b)

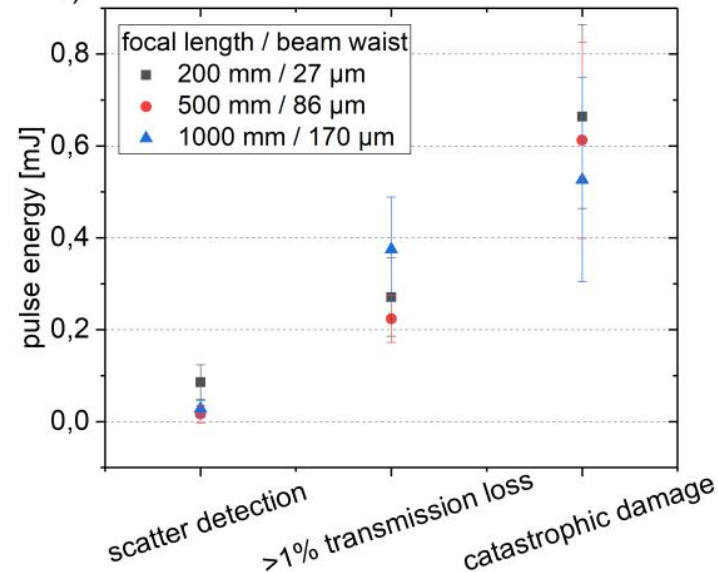

Figure 5. Damage probability for catastrophic damage for a focal length of $500 \mathrm{~mm}$ (a) and the transmittable pulse energy calculated using the linear fit of the damage probability for each damage definition and focal length (b).

The damage probability shows a partially stepped increase in dependence on the pulse energy. This behavior might indicate the existence of different defect types or a deviation in the uniformity along the fiber [6][7]. Moreover, it can be seen that the three described damage definitions can be differentiated by the transmittable pulse energies (figure $5 \mathrm{~b}$ )). In terms of laser pulse energy, the LLSC occurs approximately one magnitude below MTL or catastrophic damage. The transmittable pulse energy is by a factor 2.2 higher for catastrophic damage than for MTL. Catastrophic damage occurs at pulse energies of $(0.6 \pm 0.08) \mathrm{mJ}$ on average. In view of the focal length dependence on the transmittable pulse energy no significant behavior can be observed. Even though the catastrophic damage event shows a decreasing transmittable pulse energy with longer focal length $(0.66,0.61$ and $0.53 \mathrm{~mJ})$, the actual behavior might be different since the strong deviation of about $0.21 \mathrm{~mJ}$ does not allow a reliable statement.

For comparability with previous investigations and literature, the fluence for catastrophic damage on PMMA fibers was calculated assuming a homogeneous intensity distribution in the fiber. Under this assumption, the mean value for catastrophic damage including all focal lengths is $(0.32 \pm 0.05) \mathrm{J} / \mathrm{cm}^{2}$. In table 2 the LIDT of a corresponding PMMA preform and the threshold of a stretched PMMA waveplate investigated by Melninkaitis et al. [8] are listed to give a comparable overview of PMMA damage thresholds. In accordance to the preform measurement and the literature the damage occurred inside the bulk material and not at the interfaces.

Table 2. Laser-induced damage threshold of the polymer optical fibers measured in this study, a corresponding PMMA preform and a stretched PMMA waveplate investigated by Melninkaitis et al..

\begin{tabular}{|c|c|c|}
\hline Reference & Pulse duration [ns] & LIDT $\left[\mathrm{J} / \mathrm{cm}^{2}\right]$ \\
\hline $\begin{array}{l}\text { PMMA fiber } \\
486 \mu \mathrm{m} / 500 \mu \mathrm{m}\end{array}$ & 7.3 & 0.32 \\
\hline $\begin{array}{l}\text { Corresponding PMMA } \\
\text { preform }\end{array}$ & 8.8 & 0.57 \\
\hline $\begin{array}{l}\text { Melninkaitis et al., } \\
2006 \text { [8], stretched } \\
\text { PMMA waveplate }\end{array}$ & 3.4 & 0.46 \\
\hline
\end{tabular}


The LIDT of the PMMA preform and the stretched PMMA waveplate is by a factor 1.78 and 1.44 higher than the LIDT of the POF investigated in this study. The threshold deviation between the three references might be explained by the difference in the material and the pulse duration. Another issue might be the assumption of a homogeneous intensity distribution along the fiber. Also, it can be assumed that the fiber drawing process decreases the LIDT rather than increasing it. Therefore, a lower POF LIDT compared to the PMMA preform was expected.

\subsection{Damage morphology}

The laser-induced damage inside the POF is investigated using Laser Scanning Optical Tomography (SLOT) [9] evaluated with ImageJ [10]. This measurement allows a radial position determination of the induced damage additionally to the position along the fiber, giving information about the damage morphology for the different damage definitions. Figure 6 represents a laser-induced damage of the LLSC and the MTL event for different angles measured with SLOT after a whole R-on-1 measurement. Therefore, much higher pulse energies were transmitted possibly changing the damage morphology even though the damage shown was already induced. The images reveal, that the defect is close to or at the core-cladding-interface and is comparatively narrow in one of its dimensions. Therefore, it might be possible that this damage is induced by impurities and defects at the core-cladding-transition.
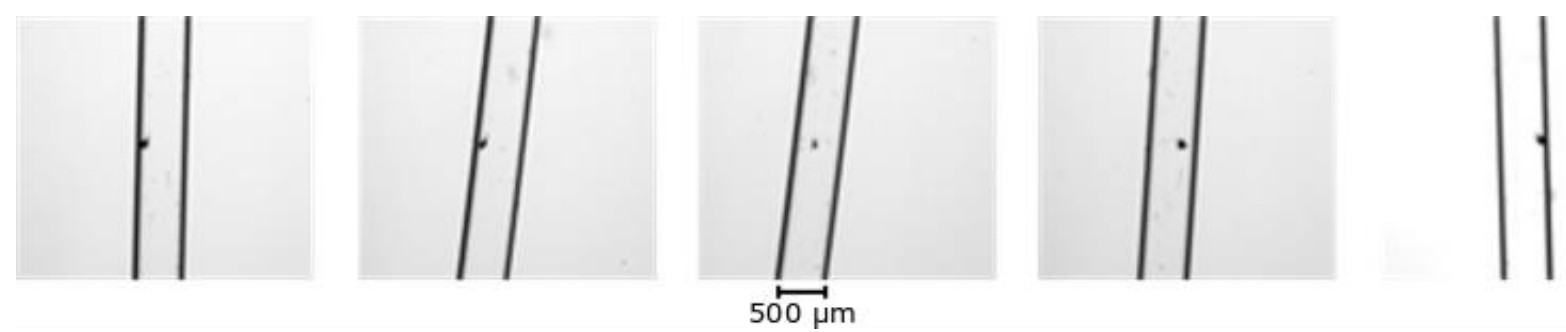

Figure 6. SLOT images of one fiber and the laser-induced damage for LLSC and MTL at different angles. Light propagated from bottom to top.

SLOT-images of catastrophic damage are shown in figure 7 and more detailed for another fiber in figure 8 . It is noted that these damages occurred within the last energy level of the R-on-1 measurement. Though, independent of the pulse number at which the damage arose the test was not stopped before all 1000 pulses were transmitted. Therefore, a change in the damage morphology by the pulses after damage detection cannot be excluded. Compared to the damage of the first two events (figure 6) the catastrophic damage affects a much wider area but is also quite narrow in a lateral plane. Moreover, it is easy to see that catastrophic damage induces a much stronger loss in transmission because of the affected area in the fiber. The comparison of the catastrophic damages of two different fibers (figure 7 and 8) shows that the damage in figure 8 seems to be closer to the fiber core center, whereas damage in figure 7 seems to be between the fiber center and the core-cladding-transition. The more detailed image of a damage (figure 8) shows that in this case the laser pulses induce a damage affecting a length of approximately $1 \mathrm{~mm}$ in the core and an oval formed area at the corecladding-transition. Apart from the radial position relative to the fiber center, both catastrophic damages have the same morphology.
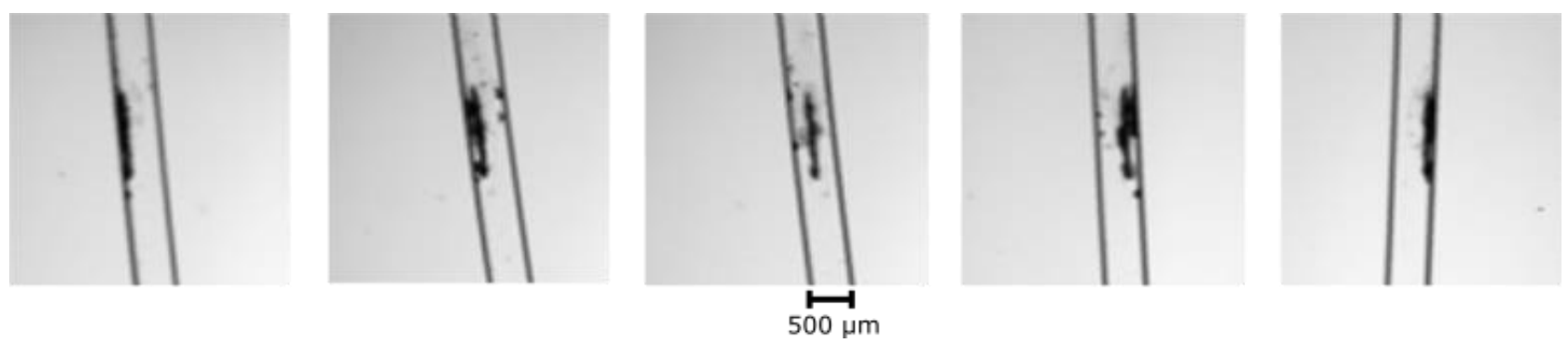

Figure 7. SLOT images of one fiber and the laser-induced damage for catastrophic damage at different angles. Damage is in the fiber core close to the core-cladding-transition. Light propagated from bottom to top. 

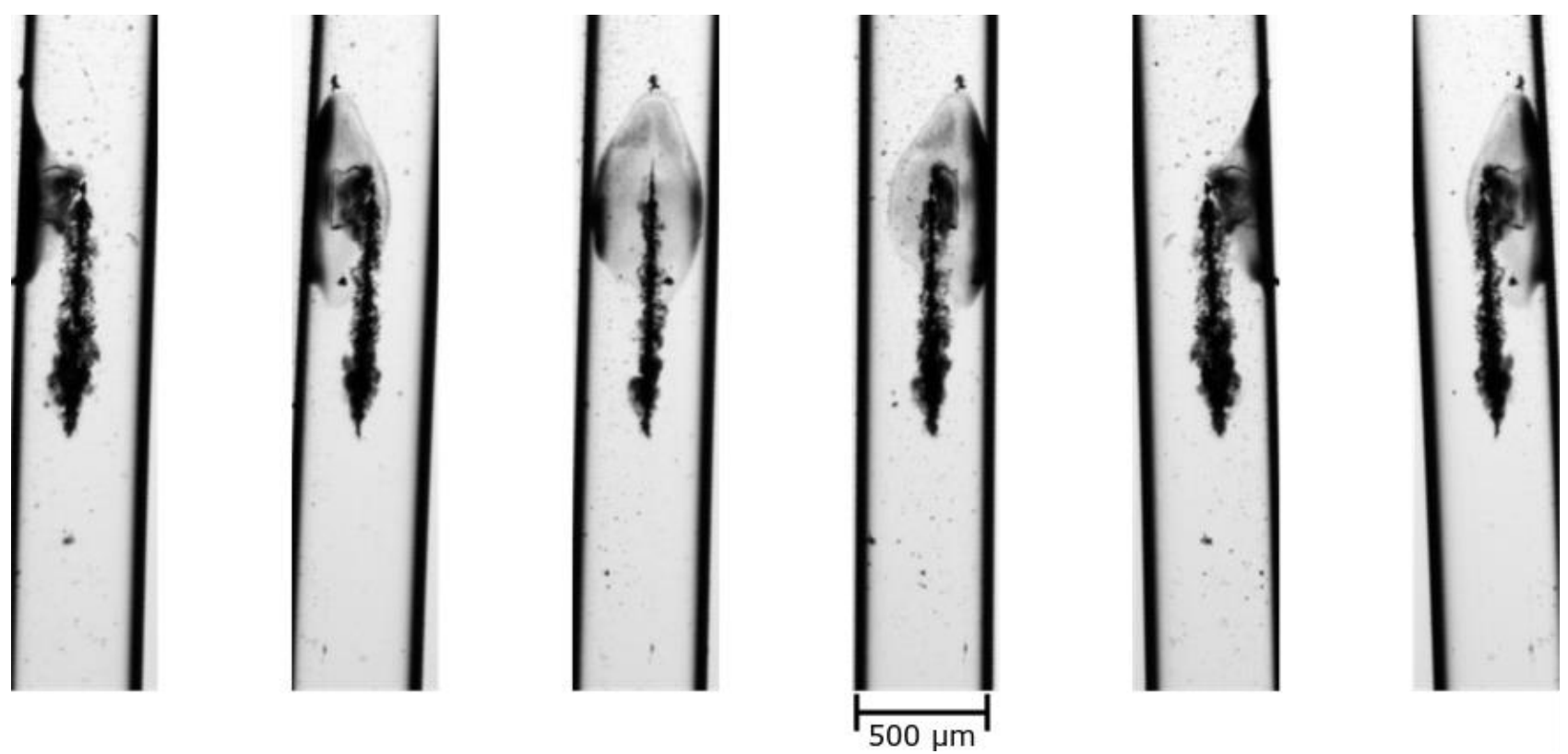

Figure 8. SLOT images of one fiber and the laser-induced damage for catastrophic damage close to the fiber core center at different angles. Light propagated from bottom to top.

\section{CONCLUSION}

The transmittable pulse energy for polymer optical fibers was studied using three different focal lengths of the focusing lens in R-on-1 measurements. In this study as well as in LID investigations at the LZH and in literature the damage for pulsed laser radiation at $532 \mathrm{~nm}$ occurred in the PMMA bulk material and not at the surfaces.

Catastrophic damage defined as a high and rapid loss in transmission occurs within the first few centimeters at pulse energies of about $0.6 \mathrm{~mJ}$, and affects both, the core material and the core-cladding-interface. After 1000 pulses propagated through the fibers a linear damage of approximately $1 \mathrm{~mm}$ length inside the fiber core with a short redirection to the fiber cladding surface can be seen. Whereas, the damage of a loss in transmission of greater than $1 \%$ forming during one energy level is a comparatively slow process happening within several hundred pulses. This damage affects mostly just a small area around the core-cladding-transition and does not seem to spread into a particular direction. Moreover, the damages occur at random positions along the fiber following no predictable behavior. Besides, SLOT images indicate that damages detected before catastrophic damage occurs are possibly induced by impurities and defects in the core-cladding-transition.

A significant dependence of the focal lengths on the transmittable pulse energy could not be observed. Solely the catastrophic damage event might indicate a lower transmittable pulse energy for a longer focal length. Though, the large measurement uncertainty prevents a reliable statement and requires further investigations.

\section{ACKNOWLEDGMENTS}

The authors thank the European Regional Development Fund for the financial support of the joint project LAPOF (contract no. ZW 685003502). We want to thank our colleagues of the Imaging and Metrology Group under the guidance of Dr.-Ing. Heiko Meyer of the Department of Industrial and Biomedical Optic of the Laser Zentrum Hannover e.V. for the measurements with the scanning laser optical tomography. 


\section{REFERENCES}

[1] Ziemann, O., Krauser, J., Zamzow, P. E. and Daum, W., [POF Handbook], Springer-Verlag, Berlin Heidelberg (2007).

[2] Gebauer, H., Jupé, M., Bataviciute, G., Ristau, D. and Kling, R., "Measurement of laser power resistance of fibers for PIV systems," Proc. SPIE 7132 (2008).

[3] Kuzyk, M. G., [Polymer Fiber Optics], CRC Press, Boca Raton (2006).

[4] Koike, Y., [Fundamentals of Plastic Optical Fibers], Wiley-VCH Verlag GmbH \& Co., Berlin (2015).

[5] Schrameyer, M., Jupé, M., Jensen, L., Ristau, D., "Algorithm for cumulative damage probability calculations in S-on-1 laser damage testing, "Proc. SPIE 8885 (2013).

[6] Porteus, J. O. and Seitel, S. C., "Absolute onset of optical surface damage using distributed defect ensembles," Applied Optics 23(21), 3796-3805 (1984).

[7] Krol, H., Gallais, L., Grèzes-Besset, C., Natoli, J.-Y., Commandré, M., "Investigation of nanoprecursors threshold distribution in laser-damage testing," Optics Communications 256(1-3), 184-189 (2005).

[8] Melninkaitis, A., Mikšys, D., Maciulevičius, M. and Sirutkaitis, V., "Laser-induced damage threshold of stretched PMMA waveplates," Proc. SPIE 6403 (2006).

[9] Lorbeer, R., Heidrich, M., Lorbeer, C., Ojeda, D., Bicker, G., Meyer, H. and Heisterkamp, A.,"Highly efficient 3D fluorescence microscopy with a scanning laser optical tomograph," Optics Express 19(6), 5419-5430 (2011).

[10] Schneider, C. A., Rasband, W. S. and Eliceiri, K. W., „NIH Image to ImageJ: 25 years of image analysis,“ Nature methods 9(7), 671-675 (2012). 